\title{
STOCHASTIC CONTROL BASED ON TIME-CHANGE TRANSFORMATIONS FOR STOCHASTIC PROCESSES WITH LÉVY NOISE
}

UDC 519.21

\author{
S. V. BODNARCHUK AND O. M. KULIK
}

\begin{abstract}
We propose a new method of stochastic control for stochastic processes with Lévy noise based on time-change transformations. Applying this method, we prove that the integral minorization condition holds for Markov processes defined by stochastic equations with Lévy noise and obtain the explicit estimates for the rate of convergence in the ergodic theorem.
\end{abstract}

\section{INTRODUCTION}

The stochastic differential equation

$$
X(x, t)=x+\int_{0}^{t} a(X(x, s)) d s+Z(t), \quad x \in \mathbb{R}^{m}, t \in \mathbb{R}^{+},
$$

is studied in the current paper where $Z$ is a Lévy process assuming values in $\mathbb{R}^{m}$. A set of sufficient conditions for the exponential ergodicity of the solution of this equation is given in the paper [1] (exponential ergodicity means the exponential rate of convergence in variation of one dimensional distributions of a solution to its unique invariant distribution, see Theorem 4.1 below). A solution of equation (1) is viewed in [1] as a Markov process with the phase space $\mathbb{R}^{m}$.

An important question related to some applied problems of statistics, modeling, etc., concerns the exact values of the constants $C_{1}$ and $C_{2}$ in the exponential estimate of the rate of convergence to the invariant distribution (see (34)). An approach developed in [1] allows one to obtain qualitative existence theorems for the corresponding estimate but quantitative results are not available in the framework of this approach. Another approach is proposed in the current paper that provides some quantitative results. Moreover, we believe that this approach may lead to generalizations of results on the exponential ergodicity for processes with infinite dimensional phase space, in particular for solutions of stochastic partial differential equations with Lévy noise.

Our approach is based on a new type of stochastic control for processes with Lévy noise, namely the control by means of time-change transformations. Theorem [2.1, the main result of the paper, contains a lower bound for the common part of distributions of two solutions of equation (11) corresponding to two different initial values. The statement of this result is given in Section 2, while Section 3 is devoted to its proof. Also, Section 3 contains a description of the construction of the stochastic control based on time-change transformations that is a key tool for the proof of Theorem 2.1. An application of the

2010 Mathematics Subject Classification. Primary 60H07; Secondary 60G51.

Key words and phrases. Lévy process, stochastic equation, stochastic control, time-change transformations. 
main result for obtaining the upper bounds of the exponential ergodicity is described in Section 4 a particular case of such an application is considered in Section 5 .

\section{Notation AND Statement of the Main REsult}

In what follows $Z(t), t \in \mathbb{R}^{+}$, denotes a Lévy process assuming values in $\mathbb{R}^{m}$; it is assumed that $Z$ has no diffusion component, that is,

$$
Z(t)=Z(0)+b t+\int_{0}^{t} \int_{|u|>1} u \nu(d s, d u)+\int_{0}^{t} \int_{|u| \leq 1} u \tilde{\nu}(d s, d u),
$$

where $b \in \mathbb{R}^{m}, \nu$ is a random Poisson point measure in $\mathbb{R}^{+} \times \mathbb{R}^{m}$ with intensity measure $d t \times \Pi(d u)$ (here $\Pi$ is the Lévy measure for $\nu)$, and $\tilde{\nu}(d s, d u)=\nu(d s, d u)-d s \Pi(d u)$ is the corresponding compensated measure.

Throughout the paper we use the following notation: $|\cdot|$ is either the absolute value of a number or the Euclidean norm of a vector; $B_{r}(x)=\{y:|y-x| \leq r\}$ is the closed ball of radius $r$ centered at $x ;\|\cdot\|$ is the matrix norm, that is, $\|C\|=\sup _{l \in \mathbb{S}^{m}}|C l| ;(\cdot, \cdot)$ is the scalar product; $\mathbb{S}^{m}=\left\{l \in \mathbb{R}^{m}:|l|=1\right\}$ is the unit sphere in $\mathbb{R}^{m}$;

$$
V_{\sigma}(l)=\left\{v \in \mathbb{R}^{m}:|(v, l)| \geq \sigma|v|\right\}, \quad l \in \mathbb{S}^{m}, \sigma \in(0,1),
$$

is a bilateral cone in $\mathbb{R}^{m}$ with an axis $l$ and gradient slope $\sigma$.

Consider the stochastic differential equation (11) and assume that $a \in C^{2}$ and that the first and second derivatives of $a$ are bounded. Put

$$
\begin{gathered}
A_{1}=\sup _{x}\|\nabla a(x)\|, \\
\nabla^{2} a(x) \otimes h=\lim _{\varepsilon \rightarrow 0} \frac{1}{\varepsilon}(\nabla a(x+\varepsilon h)-\nabla a(x)), \quad A_{2}=\sup _{x \in \mathbb{R}^{m}, l \in \mathbb{S} m}\left\|\nabla^{2} a(x) \otimes l\right\| .
\end{gathered}
$$

The distribution of the random variable $X(x, t)$ is denoted by $\mu_{x, t}$. If $\mu$ and $\kappa$ are two measures in $\mathbb{R}^{m}$, then $\mu \wedge \kappa$ denotes their common part:

$$
\mu \wedge \kappa=\int_{\mathbb{R}^{m}} \min \left(\frac{d \mu}{d \lambda}, \frac{d \kappa}{d \lambda}\right) d \lambda,
$$

where $\lambda$ is an arbitrary measure such that both $\mu$ and $\kappa$ are absolutely continuous with respect to $\lambda$ (for example, $\lambda=\mu+\kappa$ ).

For an arbitrary fixed set $\Gamma$ with $\Pi(\Gamma)<\infty$, we split the space $\mathbb{R}^{m}$ into two parts, namely

$$
\mathbb{R}^{m}=\Gamma \cup \Gamma^{c}
$$

For all $t, \varepsilon, \theta, \rho, \varrho, R_{0}, R>0, \gamma, \delta$, and $\sigma \in(0,1)$ put

$$
\begin{gathered}
\mathrm{C}=\left(\varepsilon \varrho A_{1}+2 \varepsilon R+4 \theta\right) A_{1} e^{t A_{1}}+m \varepsilon A_{1}^{2} e^{2 t A_{1}}\left(\varrho(\varepsilon+\varrho)+\frac{t A_{2} \varrho^{2}}{2}\right), \\
\Pi_{1}=\inf _{x \in \mathbb{R}^{m}} \Pi(u \in \Gamma:|a(x+u)-a(x)|>\rho,|u| \leq \varrho), \\
\Pi_{2}=\inf _{x \in \mathbb{R}^{m}, l \in \mathbb{S}^{m}} \Pi\left(u \in \Gamma:|a(x+u)-a(x)|>\rho,|u| \leq \varrho, a(x+u)-a(x) \in V_{\sigma}(l)\right), \\
\mathrm{P}_{1}=\left(1-\exp \left(-\varepsilon\left[\frac{t}{m \varepsilon}\right] \Pi_{1} e^{-\varepsilon \Pi(\Gamma)}\left(1-\frac{5 \varepsilon}{2 \theta^{2}} \int_{\Gamma^{c}}|u|^{2} \Pi(d u)\right)\right)\right) \\
\times\left(1-\exp \left(-\varepsilon\left[\frac{t}{m \varepsilon}\right] \Pi_{2} e^{-\varepsilon \Pi(\Gamma)}\left(1-\frac{5 \varepsilon}{2 \theta^{2}} \int_{\Gamma^{c}}|u|^{2} \Pi(d u)\right)\right)\right)^{m-1},
\end{gathered}
$$


where $[z]=\max \{n \in \mathbb{Z}: n \leq z\}$ is the integer part of the number $z$,

$$
\mathrm{P}_{2}=\sup _{|x| \leq R_{0}} \mathrm{P}\left(\sup _{r \leq t}|X(x, r)|>R-\frac{m \varepsilon}{2} A_{1} \varrho e^{t A_{1}}\right) .
$$

Theorem 2.1. Let $t, \varepsilon, \varkappa, \theta, \rho, \varrho, R>0, \gamma, \delta$, and $\sigma \in(0,1)$ be such that

$$
\begin{gathered}
\sqrt{m} \mathrm{C} \leq \gamma \rho \sigma^{m-1} e^{-2(m+1) t A_{1}}\left(e^{4 t A_{1}}-\sigma^{2}\right)^{1 / 2}, \\
\varkappa e^{t A_{1}} \leq \frac{1}{2} \varepsilon(1-\delta)(1-\gamma) \rho \sigma^{m-1} e^{-2(m+1) t A_{1}}\left(e^{4 t A_{1}}-\sigma^{2}\right)^{1 / 2} .
\end{gathered}
$$

Then

$$
\mu_{x, t} \wedge \mu_{y, t} \geq \delta^{m}\left(\frac{1-\gamma}{1+\gamma}\right)^{2 m}\left(\mathrm{P}_{1}-\mathrm{P}_{2}\right)
$$

for all $x$ with $|x| \leq R_{0}$ and all $y \in B_{\varkappa}(x)$.

Remark 2.1. The function $a$ satisfies the linear growth condition; that is,

$$
|a(x)| \leq A_{0}+A_{1}|x|, \quad A_{0}=|a(0)| .
$$

Thus one can estimate $\mathrm{P}_{2}$ from above by using the Gronwall lemma. However we do not do that in order to keep the main result feasible. An example of an explicit bound for $\mathrm{P}_{2}$ is given in Section 5 .

Remark 2.2. The meaning of conditions (5) and (6) is explained in Section 3.1 by the method used to obtain the main bound (18) which in turn implies (7). Note also that conditions (5) and (6) imply, in fact, assumptions (15) and (16) stated below.

Remark 2.3. It is clear that Theorem 2.1 is meaningful only if conditions (5) and (6) hold and

$$
P_{1}>P_{2}
$$

If

$$
\Pi_{1}>0, \quad \Pi_{2}>0,
$$

for some $\Gamma, \rho, \varrho$, and $\sigma$, then one can choose other parameters such that conditions (5), (6), and (8) hold. Indeed, one may assume without loss of generality that the set $\Gamma^{c}$ is bounded, whence we conclude that

$$
\int_{\Gamma^{c}}|u|^{2} \Pi(d u)<\infty
$$

Let, for example, $\theta=\varepsilon^{1 / 3}$. Then

$$
\mathrm{P}_{1} \rightarrow \mathrm{P}_{1,0}=\prod_{k=1}^{m}\left(1-\exp \left(-\frac{t}{m} \Pi_{k}\right)\right)>0, \quad \varepsilon \rightarrow 0 .
$$

On the other hand, $\mathrm{P}_{2} \rightarrow 0$ as $R \rightarrow \infty$. Choosing $R$ sufficiently large we obtain $\mathrm{P}_{1,0}>\mathrm{P}_{2}$. This means that inequalities (5) and (8) hold for sufficiently small $\varepsilon$. Now choosing $\varkappa$ and $\delta$ appropriately we prove inequality (6). 


\section{Proof of Theorem 2.1}

3.1. The construction of the stochastic control and main estimate. Let $t>0$ be fixed and let $I_{1}, \ldots, I_{m} \subset(0, t)$ be some disjoint intervals. Denote by $\tau_{j}$ the moment when the first jump occurs in the interval $I_{j}$ whose length $p_{j}$ belongs to the set $\Gamma$. If $\nu\left(I_{j} \times \Gamma\right)=0$, then the variables $\tau_{j}$ and $p_{j}$ are not defined.

Put $\mathbf{I}=\prod_{j=1}^{m} I_{j}$ and

$$
\Omega_{\mathbf{I}}=\bigcap_{j=1}^{m}\left\{\nu\left(I_{j} \times \Gamma\right)=1\right\}, \quad P_{\mathbf{I}}=\mathrm{P}\left(\cdot \mid \Omega_{\mathbf{I}}\right) .
$$

In the set $\Omega_{\mathbf{I}}$, the solution $X(x, t)$ of equation (2) is a measurable function of the variables

$$
x, \quad \boldsymbol{\tau}=\left\{\tau_{j}\right\}_{j=1}^{m}, \quad \mathbf{p}=\left\{p_{j}\right\}_{j=1}^{m}, \quad \nu_{\mathbf{I}}=\left.\nu\right|_{[0, t] \times \mathbb{R}^{m} \backslash\left(\bigcup_{j=1}^{m} I_{j}\right) \times \Gamma} .
$$

Note that $\nu_{\mathbf{I}}$ assumes its values in the space of configurations $\mathfrak{C}$ in $\mathbb{R}^{+} \times\left(\mathbb{R}^{m} \backslash\{0\}\right)$ that can be viewed as a complete separable metric space; see [2]. Recall that a configuration is a locally bounded set, by definition. The elements $\boldsymbol{\tau}, \mathbf{p}$, and $\nu_{\mathbf{I}}$ are jointly independent with respect to $P_{\mathbf{I}}$. Moreover, $\boldsymbol{\tau}$ is uniformly distributed in $\mathbf{I}$ and components of the vector $\mathbf{p}$ are independent identically distributed random variables with the distribution

$$
\Pi_{\Gamma}=\frac{\Pi(\cdot \cap \Gamma)}{\Pi(\Gamma)} .
$$

In what follows let

$$
X(x, t)=F\left(x, t, \boldsymbol{\tau}, \mathbf{p}, \nu_{\mathbf{I}}\right),
$$

where $F$ is a measurable function assuming values in $\mathbb{R}^{m}$. Let $\hat{\mathbf{I}}=\prod_{j=1}^{m} \hat{I}_{j}$, where $\hat{I}_{j}$ is the interval whose center coincides with that of $I_{j}$ and whose length is $\delta\left|I_{j}\right|$. Given some $\delta \in(0,1)$ and some set $\Upsilon_{\mathbf{I}} \subset\left(\mathbb{R}^{m}\right)^{m} \times \mathfrak{C}$, our aim is to construct a function

$$
K: B_{\varkappa}(x) \times \hat{\mathbf{I}} \times \Upsilon_{\mathbf{I}} \rightarrow \mathbf{I}
$$

such that

$$
\begin{gathered}
F\left(y, t, K\left(y, \boldsymbol{\tau}, \mathbf{p}, \nu_{\mathbf{I}}\right), \mathbf{p}, \nu_{\mathbf{I}}\right)=F\left(x, t, \boldsymbol{\tau}, \mathbf{p}, \nu_{\mathbf{I}}\right), \\
y \in B_{\varkappa}(x), \quad \boldsymbol{\tau} \in \hat{\mathbf{I}}, \quad\left(\mathbf{p}, \nu_{\mathbf{I}}\right) \in \Upsilon_{\mathbf{I}} .
\end{gathered}
$$

Roughly speaking, the mapping $K$ defines a transformation of the set of jump moments $\boldsymbol{\tau}=\left\{\tau_{j}\right\}_{j=1}^{m}$ that transforms the value at time $t$ of the solution of equation (11) with the initial values $y$ in the value at time $t$ of the solution with the initial value $x$. Note that the time variable $\boldsymbol{\tau}$ changes under this transformation, while $\mathbf{p}$ and $\nu_{\mathbf{I}}$ play the role of parameters. The function $K$ defines the time-change transformation for the stochastic control discussed in Section 1 Such a control is defined in the subset

$$
\{\boldsymbol{\tau} \in \hat{\mathbf{I}}\} \cap \Xi_{\mathbf{I}}
$$

of the probability space $\Omega$. Here

$$
\Xi_{\mathbf{I}}=\Omega_{\mathbf{I}} \cap\left\{\left(\mathbf{p}, \nu_{\mathbf{I}}\right) \in \Upsilon_{\mathbf{I}}\right\}
$$

If $\omega \in \Omega$ is identified with a configuration of a Poisson random measure $\nu$, then the points of $\{\boldsymbol{\tau} \in \hat{\mathbf{I}}\} \cap \Xi_{\mathbf{I}}$ are naturally considered as admissible configurations for a stochastic control based on the time-change transformation. 
The construction of the control $K$ is based on the differential properties of the solution $X(x, t)$ of equation (1). Propositions 4.1 and 4.2 of the paper [2] imply that this solution as a function of the variable $\boldsymbol{\tau} \in \mathbf{I}$ has the Sobolev derivative

$$
\nabla_{\boldsymbol{\tau}} X(x, t)=\left(\frac{\partial}{\partial \tau_{1}} X(x, t), \ldots, \frac{\partial}{\partial \tau_{m}} X(x, t)\right)
$$

for all $x$ and $\mathbf{p}$ and for almost all realizations of the random point measure $\nu_{\mathbf{I}}$.

Moreover

$$
\frac{\partial}{\partial \tau_{j}} X(x, t)=\mathcal{E}_{\tau_{j}}^{t} \Delta\left(X\left(x, \tau_{j}-\right), p_{j}\right), \quad j=1, \ldots, m
$$

where

$$
\Delta(x, p)=a(x+p)-a(x)
$$

and where the stochastic exponent $\left\{\mathcal{E}_{s}^{v}, 0 \leq s \leq r\right\}$ is a matrix-valued process defined by the family of equations

$$
\mathcal{E}_{s}^{r}=I_{\mathbb{R}^{m}}+\int_{s}^{r} \nabla a(X(x, v)) \mathcal{E}_{s}^{v} d v, \quad r \in[s, \infty) .
$$

Note that $\nabla_{x} X(x, t)=\mathcal{E}_{0}^{t}$, whence the bound

$$
\left\|\nabla_{x} X(x, t)\right\| \leq e^{t A_{1}}
$$

follows. Let $\varepsilon=\min _{j}\left|I_{j}\right|$. Similarly to (10), we put

$$
\nabla \boldsymbol{\tau} X(x, t)=G\left(x, t, \boldsymbol{\tau}, \mathbf{p}, \nu_{\mathbf{I}}\right) .
$$

Given $x, t$, and some $\gamma \in(0,1)$, assume that one can find a set $\Upsilon_{\mathbf{I}}$ and a measurable function $H$ of the variables $\mathbf{p}$ and $\nu_{\mathbf{I}}$ that assumes values in $\mathbb{R}^{m \times m}$ and such that

$$
\sup _{\boldsymbol{\tau} \in \mathbf{I}}\left\|G\left(x, t, \boldsymbol{\tau}, \mathbf{p}, \nu_{\mathbf{I}}\right)-H\left(\mathbf{p}, \nu_{\mathbf{I}}\right)\right\| \leq \gamma\left\|\left[H\left(\mathbf{p}, \nu_{\mathbf{I}}\right)\right]^{-1}\right\|^{-1} .
$$

By the implicit function theorem (see Theorem B.1 below), if $\varkappa$ and $\delta$ are arbitrary numbers such that

$$
\varkappa e^{t A_{1}}\left\|\left[H\left(\mathbf{p}, \nu_{\mathbf{I}}\right)\right]^{-1}\right\| \leq \frac{1}{2} \varepsilon(1-\delta)(1-\gamma),
$$

then a function $K$ exists such that relation (11) holds. Moreover, the function $K$ is uniquely defined by relation (11).

Further reasoning is based on the change of variables formula for Lipshitz mappings (see [3, Chapter 3]) and on the following properties of implicit functions that, given fixed $y, \mathbf{p}$, and $\nu_{\mathbf{I}}, K_{y, \mathbf{p}, \nu_{\mathbf{I}}}=K\left(y, \cdot, \mathbf{p}, \nu_{\mathbf{I}}\right)$ is a Lipshitz injective mapping (see Appendix B). Then the image of the restriction of the Lebesgue measure on $\hat{\mathbf{I}}$ has density under this transformation, and the density is given by

$$
\left[\operatorname{det} \nabla K_{y, \mathbf{p}, \nu_{\mathbf{I}}}\left(K_{y, \mathbf{p}, \nu_{\mathbf{I}}}^{-1}(z)\right)\right]^{-1} \mathbb{1}_{z \in K_{y, \mathbf{p}, \nu_{\mathbf{I}}}(\hat{\mathbf{I}})}
$$

in view of the change of variables formula. Thus the common part of the conditional distribution of

$$
F\left(y, t, \boldsymbol{\tau}, \mathbf{p}, \nu_{\mathbf{I}}\right)
$$

and the conditional distribution of $F\left(x, t, \boldsymbol{\tau}, \mathbf{p}, \nu_{\mathbf{I}}\right)$ is not less than

$$
\begin{aligned}
& \left(\prod_{j=1}^{m}\left|I_{j}\right|\right)^{-1} \int_{K_{y, \mathbf{p}, \nu_{\mathbf{I}}}(\hat{\mathbf{I}})}\left(\left|\operatorname{det} \nabla K_{y, \mathbf{p}, \nu_{\mathbf{I}}}\left(K_{y, \mathbf{p}, \nu_{\mathbf{I}}}^{-1}(z)\right)\right|^{-1} \wedge 1\right) d z \\
& \quad \geq\left(\prod_{j=1}^{m}\left|I_{j}\right|\right)^{-1}\left(\inf _{z \in \mathbf{I}}\left|\operatorname{det} \nabla K_{y, \mathbf{p}, \nu_{\mathbf{I}}}(z)\right|^{-1} \wedge 1\right) \lambda^{m}\left(K_{y, \mathbf{p}, \nu_{\mathbf{I}}}(\hat{\mathbf{I}})\right)
\end{aligned}
$$


if condition $\left(\mathbf{p}, \nu_{\mathbf{I}}\right)$ holds (see statement 1 of Lemma A.2 below). On the other hand,

$$
\int_{K_{y, \mathbf{p}, \nu_{\mathbf{I}}}(\hat{\mathbf{I}})}\left|\operatorname{det} \nabla K_{y, \mathbf{p}, \nu_{\mathbf{I}}}\left(K_{y, \mathbf{p}, \nu_{\mathbf{I}}}^{-1}(z)\right)\right|^{-1} d z=\prod_{j=1}^{m}\left|\hat{I}_{j}\right|=\delta^{m} \prod_{j=1}^{m}\left|I_{j}\right|
$$

and hence the common part of the conditional distribution of $F\left(y, t, \boldsymbol{\tau}, \mathbf{p}, \nu_{\mathbf{I}}\right)$ and the conditional distribution of

$$
F\left(x, t, \boldsymbol{\tau}, \mathbf{p}, \nu_{\mathbf{I}}\right)
$$

is not less than

$$
\delta^{m} \frac{\inf _{z \in \hat{\mathbf{I}}}\left|\operatorname{det} \nabla K_{y, \mathbf{p}, \nu_{\mathbf{I}}}(z)\right|}{1 \vee \sup _{z \in \hat{\mathbf{I}}}\left|\operatorname{det} \nabla K_{y, \mathbf{p}, \nu_{\mathbf{I}}}(z)\right|} .
$$

Taking into account bound (45), we derive from this that this common part is not less than $\delta^{m}((1-\gamma) /(1+\gamma))^{2 m}$.

Let $\Xi \subset \Xi_{\mathbf{I}}$ be a random event of the form

$$
\Xi=\Omega_{\mathbf{I}} \cap\left\{\left(\mathbf{p}, \nu_{\mathbf{I}}\right) \in \Upsilon\right\},
$$

where $\Upsilon \subset \Upsilon_{\mathbf{I}}$ is a measurable set.

Let $\mu_{x, t, \Xi}$ be the distribution of the restriction of $X(x, t)$ to the set $\Xi$. Then the above estimates and statement 3 of Lemma A.2 imply the main estimate

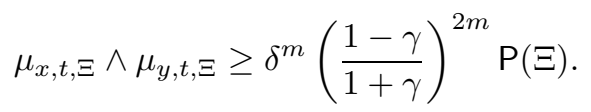

3.2. The set $\Upsilon_{\mathbf{I}}$ and estimates for the gradient. The reasoning in the preceding section is essentially based on the assumption (15). In this section, we construct a set $\Upsilon_{\mathbf{I}}$ in such a way that assumption (15) holds.

First we introduce an auxiliary notation. Let $\hat{\tau}_{j}$ be the center of the interval $I_{j}$, $j=1, \ldots, m$, and let $\hat{\boldsymbol{\tau}}=\left\{\hat{\tau}_{j}\right\}_{j=1}^{m}$. In the set $\Omega_{\mathbf{I}}$, put

$$
\hat{X}(x, r)=F\left(x, r, \hat{\boldsymbol{\tau}}, \mathbf{p}, \nu_{\mathbf{I}}\right), \quad H\left(\mathbf{p}, \nu_{\mathbf{I}}\right)=G\left(x, t, \hat{\boldsymbol{\tau}}, \mathbf{p}, \nu_{\mathbf{I}}\right) ;
$$

see (10) and (14). In other words, $\hat{X}$ is a solution of equation (11) obtained from the process $Z$ by using the jump moments $\hat{\tau}_{1}, \ldots, \hat{\tau}_{m}$ instead of $\tau_{1}, \ldots, \tau_{m}$ (the lengths of the jumps remain the same). We also denote by $\hat{\mathcal{E}}_{s}^{r}$ the solution of equation (13) where $\hat{X}$ is used instead of $X$. Then the rows of the matrix $H\left(\mathbf{p}, \nu_{\mathbf{I}}\right)$ are determined by the right-hand side of (12) where $\hat{\tau}_{j}, \hat{X}$, and $\hat{\mathcal{E}}$ are substituted for $\tau_{j}, X$, and, $\mathcal{E}$, respectively.

To satisfy condition (15), we introduce two sets of restrictions. The first of them allows one to control the norm of the inverse matrix to $H\left(\mathbf{p}, \nu_{\mathbf{I}}\right)$. First, we choose measurable functions

$$
\ell_{j}:\left(\mathbb{R}^{m}\right)^{j} \rightarrow \mathbb{S}^{m}, \quad j=1, \ldots, m-1,
$$

such that

$$
\ell_{j}\left(x_{1}, \ldots x_{j}\right) \perp \operatorname{span}\left(x_{1}, \ldots, x_{j}\right)
$$

for all $j \leq m-1$ and $x_{1}, \ldots, x_{j} \in \mathbb{R}^{m}$. Then we put

$$
\begin{aligned}
l_{j} & =l_{j}\left(\mathbf{p}, \nu_{\mathbf{I}}\right) \\
& =c_{j}\left(\left[\hat{\mathcal{E}}_{0}^{\hat{\gamma}_{j}}\right]^{-1}\right)^{*} \ell_{j-1}\left(\left[\hat{\mathcal{E}}_{0}^{\hat{\tau}_{1}}\right]^{-1} \Delta\left(\hat{X}\left(x, \hat{\tau}_{1}-\right)\right), \ldots,\left[\hat{\mathcal{E}}_{0}^{\hat{\tau}_{j-1}}\right]^{-1} \Delta\left(\hat{X}\left(x, \hat{\tau}_{j-1}-\right)\right)\right),
\end{aligned}
$$


$j=2, \ldots, m$, where $c_{j}=c_{j}\left(\mathbf{p}, \nu_{\mathbf{I}}\right)>0$ are such that $l_{j} \in \mathbb{S}^{m}$. Another restriction we impose is that, given $\rho>0$ and $\sigma \in(0,1)$,

$$
\begin{gathered}
\left|\Delta\left(\hat{X}\left(x, \hat{\tau}_{j}-\right), p_{j}\right)\right| \geq \rho, \quad j=1, \ldots, m, \\
\Delta\left(\hat{X}\left(x, \hat{\tau}_{j}-\right), p_{j}\right) \in V_{\sigma}\left(l_{j}\right), \quad j=2, \ldots, m,
\end{gathered}
$$

in the set $\Upsilon_{\mathbf{I}}$.

Lemma 3.1. Let condition (20) hold. Then

$$
\left\|\left[H\left(\mathbf{p}, \nu_{\mathbf{I}}\right)\right]^{-1}\right\| \leq \frac{e^{2(m+1) t A_{1}}}{\rho \sigma^{m}}\left(\frac{e^{4 t A_{1}}}{\sigma^{2}}-1\right)^{-1 / 2} .
$$

Proof. The matrix $H\left(\mathbf{p}, \nu_{\mathbf{I}}\right)$ admits the representation

$$
H\left(\mathbf{p}, \nu_{\mathbf{I}}\right)=\hat{\mathcal{E}}_{0}^{t} Q\left(\mathbf{p}, \nu_{\mathbf{I}}\right),
$$

where the rows of the matrix $Q\left(\mathbf{p}, \nu_{\mathbf{I}}\right)$ are such that

$$
Q_{j}=\left[\hat{\mathcal{E}}_{0}^{\hat{\tau}_{j}}\right]^{-1} \Delta\left(\hat{X}\left(x, \hat{\tau}_{j}-\right)\right), \quad j=1, \ldots, m .
$$

It is known that $\left\|\left[\hat{\mathcal{E}}_{0}^{t}\right]^{-1}\right\| \leq e^{t A_{1}}$, whence we conclude that the result desired follows from the bound

$$
\left\|\left[Q\left(\mathbf{p}, \nu_{\mathbf{I}}\right)\right]^{-1}\right\| \leq \frac{e^{(2 m+1) t A_{1}}}{\rho \sigma^{m}}\left(\frac{e^{4 t A_{1}}}{\sigma^{2}}-1\right)^{-1 / 2} .
$$

To prove the latter bound we choose an appropriate orthonormal basis $\left\{e_{j}\right\}$ in $\mathbb{R}^{m}$. Namely, let $e_{1}$ be a vector collinear to $\left[\hat{\mathcal{E}}_{0}^{\hat{\tau}_{j}}\right]^{-1} \Delta\left(\hat{X}\left(x, \hat{\tau}_{j}-\right)\right)$, while the other vectors $e_{j}$, $j \geq 2$, are such that

$$
\ell_{j-1}\left(\left[\hat{\mathcal{E}}_{0}^{\hat{\tau}_{1}}\right]^{-1} \Delta\left(\hat{X}\left(x, \hat{\tau}_{1}-\right)\right), \ldots,\left[\hat{\mathcal{E}}_{0}^{\hat{\tau}_{j-1}}\right]^{-1} \Delta\left(\hat{X}\left(x, \hat{\tau}_{j-1}-\right)\right)\right) .
$$

Then $Q\left(\mathbf{p}, \nu_{\mathbf{I}}\right)$ is a lower-triangle matrix in this basis which helps to construct an iterative procedure to estimate the norm of its inverse matrix. First, we rewrite the matrix $Q\left(\mathbf{p}, \nu_{\mathbf{I}}\right)$ in block form as

$$
Q\left(\mathbf{p}, \nu_{\mathbf{I}}\right)=: C_{m}=\left(\begin{array}{cc}
C_{m-1} & 0 \\
v_{m} & b_{m}
\end{array}\right),
$$

where $C_{m-1}$ is the restriction of $C_{m}$ to the subspace generated by $e_{1}, \ldots, e_{m-1}$ and where $v_{m}$ is the projection of $Q_{m}$ (the $m$-th row of the matrix $C_{m}$ ) to this subspace, $b_{m}=\left(Q_{m}, e_{m}\right)$. Then

$$
\begin{gathered}
C_{m}^{-1}=\left(\begin{array}{cc}
C_{m-1}^{-1} & 0 \\
-b_{m}^{-1} C_{m-1}^{-1} v_{m} & b_{m}^{-1}
\end{array}\right), \\
\left\|C_{m}^{-1}\right\|^{2} \leq\left\|C_{m-1}^{-1}\right\|^{2}+\left\|C_{m-1}^{-1}\right\|^{2}\left(\left|v_{m}\right| / b_{m}\right)^{2}+\left(1 / b_{m}^{2}\right) .
\end{gathered}
$$

The estimates for the restrictions $C_{j}$ of the matrix $C_{m}$ to the subspaces generated by $e_{1}, \ldots, e_{j}$ are given similarly:

$$
\left\|C_{j}^{-1}\right\|^{2} \leq\left\|C_{j-1}^{-1}\right\|^{2}+\left\|C_{j-1}^{-1}\right\|^{2}\left(\left|v_{j}\right| / b_{j}\right)^{2}+\left(1 / b_{j}^{2}\right), \quad j \geq 2 .
$$

Finally, we have for $j=1$ that

$$
\left\|C_{1}^{-1}\right\|^{2}=\left(1 / b_{1}^{2}\right)
$$

By construction,

$$
\left|b_{1}\right|=\left|\left[\hat{\mathcal{E}}_{0}^{\hat{\tau}_{1}}\right]^{-1} \Delta_{1}\right| \geq e^{-t A_{1}}\left|\Delta_{1}\right| \geq e^{-t A_{1}} \rho,
$$


where $\Delta_{j}=\Delta\left(\hat{X}\left(x, \hat{\tau}_{j}-\right), p_{j}\right)$ and where we applied the first assumption in (20). Similarly, we obtain

$$
\left|\left[\hat{\mathcal{E}}_{0}^{\hat{\tau}_{j}}\right]^{-1} \Delta_{j}\right| \geq e^{-t A_{1}} \rho
$$

for $j=2, \ldots, m$. Moreover, the second assumption in (20) yields

$$
\left|b_{j}\right|=\left|\left(\left[\hat{\mathcal{E}}_{0}^{\hat{\tau}_{j}}\right]^{-1} \Delta_{j}, e_{j}\right)\right|=c_{j}^{-1}\left|\left(\Delta_{j}, l_{j}\right)\right| \geq \frac{\sigma}{c_{j}}\left|\Delta_{j}\right| \geq \sigma e^{-t A_{1}}\left|\Delta_{j}\right|,
$$

where we used the inequality $c_{j} \leq e^{t A_{1}}$. This implies that

$$
\begin{gathered}
\left|b_{j}\right| \geq \sigma e^{-t A_{1}} \rho, \quad\left|v_{j}\right|^{2}=\left|\left[\hat{\mathcal{E}}_{0}^{\hat{\gamma}_{j}}\right]^{-1} \Delta_{j}\right|^{2}-\left|b_{j}\right|^{2} \leq e^{2 t A_{1}}\left|\Delta_{j}\right|^{2}-\sigma^{2} e^{-2 t A_{1}}\left|\Delta_{j}\right|^{2} \\
\left(\left|v_{j}\right| / b_{j}\right)^{2} \leq e^{4 t A_{1}} \sigma^{-2}-1,
\end{gathered}
$$

whence

$$
\left\|C_{j}^{-1}\right\|^{2} \leq e^{4 t A_{1}} \sigma^{-2}\left\|C_{j-1}^{-1}\right\|^{2}+e^{2 t A_{1}} \sigma^{-2} \rho^{-2} .
$$

Using the induction we prove the estimate

$$
\left\|C_{j}^{-1}\right\|^{2} \leq \frac{e^{2 t A_{1}}}{\rho^{2} \sigma^{2}}\left[1+\cdots+\frac{e^{4 t A_{1}(j-1)}}{\sigma^{2(j-1)}}\right] \leq \frac{e^{(4 j+2) t A_{1}}}{\rho^{2} \sigma^{2 j}}\left(\frac{e^{4 t A_{1}}}{\sigma^{2}}-1\right)^{-1}, \quad j=1, \ldots, m,
$$

which implies (21) if $j=m$.

The second set of restrictions mentioned above allows us to control the deviation

$$
\left\|G\left(x, t, \boldsymbol{\tau}, \mathbf{p}, \nu_{\mathbf{I}}\right)-H\left(\mathbf{p}, \nu_{\mathbf{I}}\right)\right\| .
$$

The partition (4) generates a representation of the Lévy process (11) in the form of two independent components, $Z=Z_{1}+Z_{2}$, where

$$
Z_{2}(t)=\int_{0}^{t} \int_{\Gamma^{c}} u \tilde{\nu}(d s, d u)
$$

We require that

$$
\sup _{s \in I_{j}}\left|Z_{2}(s)-Z_{2}\left(\hat{\tau}_{j}\right)\right| \leq \theta, \quad j=1, \ldots, m,
$$

for a given $\theta>0$. Moreover, we require that

$$
\begin{gathered}
\left|p_{j}\right| \leq \varrho, \quad j=1, \ldots, m, \\
\sup _{r \leq t}|\hat{X}(x, r)| \leq R
\end{gathered}
$$

for given $\varrho>0$ and $R>1$. Recall that $\varepsilon=\min _{j}\left|I_{j}\right|$.

Lemma 3.2. Let conditions (22), (23), and (24) hold. Then

$$
\sup _{\boldsymbol{\tau} \in \mathbf{I}}\left\|G\left(x, t, \boldsymbol{\tau}, \mathbf{p}, \nu_{\mathbf{I}}\right)-H\left(\mathbf{p}, \nu_{\mathbf{I}}\right)\right\| \leq \sqrt{m} \mathrm{C} .
$$

The constant $\mathrm{C}$ is defined in Section 2 .

Proof. The rows of the matrix $G\left(x, t, \boldsymbol{\tau}, \mathbf{p}, \nu_{\mathbf{I}}\right)$ are such that

$$
\mathcal{E}_{\tau_{j}}^{t} \Delta\left(X\left(x, \tau_{j}-\right), p_{j}\right), \quad j=1, \ldots, m .
$$

The rows of the matrix $H\left(\mathbf{p}, \nu_{\mathbf{I}}\right)$ are written similarly if one substitutes $\hat{\tau}, \hat{X}$, and $\hat{\mathcal{E}}$ for $\tau, X$, and $\mathcal{E}$, respectively. This means that one only needs to prove that the inequalities

$$
\left|\mathcal{E}_{\tau_{j}}^{t} \Delta\left(X\left(x, \tau_{j}-\right), p_{j}\right)-\hat{\mathcal{E}}_{\hat{\tau}_{j}}^{t} \Delta\left(\hat{X}\left(x, \hat{\tau}_{j}-\right), p_{j}\right)\right| \leq \mathrm{C}, \quad j=1, \ldots, m,
$$

hold for arbitrary $\left(\tau_{1}, \ldots, \tau_{m}\right) \in \mathbf{I}$. 
It is clear that:

1) $\left\|\mathcal{E}_{s}^{t}\right\| \leq e^{t A_{1}}, s \leq t$

2) $|\Delta(x, p)|=|a(x+p)-a(x)| \leq A_{1} \varrho$; we used (23) here.

3) $\left\|\mathcal{E}_{s}^{t}-\mathcal{E}_{s^{\prime}}^{t}\right\| \leq\left|s-s^{\prime}\right| A_{1} e^{t A_{1}}, s, s^{\prime} \leq t$.

4) If additionally

$$
\sup _{r \leq t}|X(x, r)| \leq R^{\prime}
$$

then

$$
\begin{aligned}
\left|X(x, s)-X\left(x, s^{\prime}\right)\right| & =\left|\int_{s \wedge s^{\prime}}^{s \vee s^{\prime}} a(X(x, r)) d r+Z(s)-Z\left(s^{\prime}\right)\right| \\
& \leq R^{\prime} \varepsilon+2 \theta, \quad s, s^{\prime} \in I_{j}
\end{aligned}
$$

we used bounds (22) here.

5) $\left|\Delta(x, p)-\Delta\left(x^{\prime}, p\right)\right| \leq\left|a(x+p)-a\left(x^{\prime}+p\right)\right|-\left|a(x)-a\left(x^{\prime}\right)\right| \leq 2 A_{1}\left|x-x^{\prime}\right|$.

It follows from 1$)-5)$ that

$$
\left|\mathcal{E}_{\tau_{j}}^{t} \Delta\left(X\left(x, \tau_{j}-\right), p_{j}\right)-\mathcal{E}_{\hat{\tau}_{j}}^{t} \Delta\left(X\left(x, \hat{\tau}_{j}-\right), p_{j}\right)\right| \leq A_{1} e^{t A_{1}}\left(\varrho A_{1} \varepsilon+2 R^{\prime} \varepsilon+4 \theta\right)
$$

under additional assumption (26).

Similarly to (12), we obtain

$$
\frac{\partial}{\partial \tau_{j}} X(x, r)=\mathcal{E}_{\tau_{j}}^{r} \Delta\left(X\left(x, \tau_{j}-\right), p_{j}\right) \mathbb{1}_{r \geq \tau_{j}}, \quad j=1, \ldots, m,
$$

for all $r$ which together with bounds 1) and 2) implies that

$$
\left|\frac{\partial}{\partial \tau_{j}} X(x, r)\right| \leq A_{1} \varrho e^{t A_{1}}, \quad j=1, \ldots, m .
$$

Hence

$$
|X(x, r)-\hat{X}(x, r)| \leq \frac{1}{2} A_{1} \varrho e^{t A_{1}} \sum_{j=1}^{m}\left|I_{j}\right|,
$$

where we used the inequality $\left|\tau_{j}-\hat{\tau}_{j}\right| \leq\left|I_{j}\right| / 2$.

Note that assumption (26) holds with

$$
R^{\prime}=R+\frac{m \varepsilon}{2} A_{1} \varrho e^{t A_{1}}
$$

in view of (28) and (24), whence

$$
\begin{aligned}
& \left|\mathcal{E}_{\tau_{j}}^{t} \Delta\left(X\left(x, \tau_{j}-\right), p_{j}\right)-\mathcal{E}_{\hat{\tau}_{j}}^{t} \Delta\left(X\left(x, \hat{\tau}_{j}-\right), p_{j}\right)\right| \\
& \quad \leq A_{1} e^{t A_{1}}\left(A_{1} \varrho \varepsilon+2 R \varepsilon+m \varepsilon^{2} A_{1} \varrho e^{t A_{1}}+4 \theta\right) .
\end{aligned}
$$

Following the lines of the proof of Propositions 4.1 and 4.2 of [2], one can show that the stochastic exponent $\mathcal{E}_{s}^{r}$, as a function of variables $\tau_{j}, j=1, \ldots, m$, possesses the Sobolev derivatives in I. Moreover, the derivative $\mathcal{G}_{s, j}^{r}=\left(\partial / \partial \tau_{j}\right) \mathcal{E}_{s}^{r}$ is a solution of the equation

$$
\mathcal{G}_{s, j}^{r}=\int_{s}^{r}\left(\nabla^{2} a(X(x, v)) \otimes\left[\mathcal{E}_{\tau_{j}}^{v} \Delta\left(X\left(x, \tau_{j}-\right), p_{j}\right)\right]\right) \mathcal{E}_{s}^{v} \mathbb{1}_{v \geq \tau_{j}} d v+\int_{s}^{r} \nabla a(X(x, v)) \mathcal{G}_{s, j}^{v} d v
$$


$r \in[s, \infty)$. Therefore

$$
\begin{gathered}
\frac{\partial}{\partial \tau_{j}} \mathcal{E}_{s}^{r}=\int_{s}^{r} \mathcal{E}_{v}^{r}\left(\nabla^{2} a(X(x, v)) \otimes\left[\mathcal{E}_{\tau_{j}}^{v} \Delta\left(X\left(x, \tau_{j}-\right), p_{j}\right)\right]\right) \mathcal{E}_{s}^{v} \mathbb{1}_{v \geq \tau_{j}} d v \\
\left|\frac{\partial}{\partial \tau_{j}} \mathcal{E}_{s}^{r}\right| \leq t A_{1} A_{2} \varrho e^{2 t A_{1}}, \quad j=1, \ldots, m,
\end{gathered}
$$

and thus

$$
\left|\mathcal{E}_{r}^{t}-\hat{\mathcal{E}}_{r}^{t}\right| \leq \frac{1}{2} t A_{1} A_{2} \varrho e^{2 t A_{1}} \sum_{j=1}^{m}\left|I_{j}\right| .
$$

Applying (28) and (31) with $r=\hat{\tau}_{j}$ together with bounds 1), 2), and 5) we obtain

$$
\left|\mathcal{E}_{\hat{\tau}_{j}}^{t} \Delta\left(X\left(x, \hat{\tau}_{j}-\right), p_{j}\right)-\hat{\mathcal{E}}_{\hat{\tau}_{j}}^{t} \Delta\left(\hat{X}\left(x, \hat{\tau}_{j}-\right), p_{j}\right)\right| \leq m \varepsilon A_{1}^{2} e^{2 t A_{1}}\left(\varrho^{2}+\frac{t A_{2} \varrho^{2}}{2}\right) .
$$

The latter inequality and (29) imply (25).

3.3. Completion of the proof of Theorem 2.1. Put $n=[t /(m \varepsilon)]$ and

$$
I^{(k)}=((k-1) \varepsilon, k \varepsilon), \quad k=1, \ldots, n m .
$$

Similarly to Section 3.1, let $\tau^{(k)}$ be the moment that the first jump occurred in the interval $I^{(k)}$ such that the length of the jump $p^{(k)}$ belongs to the set $\Gamma$. Also let $\hat{\tau}^{(k)}$ be the middle point of the interval $I^{(k)}$.

Now we define the stopping times $v_{j}, j=1, \ldots, m$. Let $v_{1}$ be the minimal $k \leq m n$ such that

$$
\begin{gathered}
\left\{\nu\left(I^{(k)} \times \Gamma\right)=1\right\}, \quad\left|\Delta\left(X\left(x, \hat{\tau}^{(k)}-\right), p^{(k)}\right)\right| \geq \rho, \\
\sup _{s \in I^{(k)}}\left|Z_{2}(s)-Z_{2}\left(\hat{\tau}^{(k)}\right)\right| \leq \theta, \quad\left|p^{(k)}\right| \leq \varrho .
\end{gathered}
$$

We put $v_{1}=+\infty$ if such a value $k$ does not exist.

For $j=2, \ldots, m$, denote by $\hat{X}$ the solution of equation (1), where the jump moments $\tau^{\left(v_{1}\right)}, \ldots, \tau^{\left(v_{j-1}\right)}$ related to the process $Z$ are replaced with $\hat{\tau}^{\left(v_{1}\right)}, \ldots, \hat{\tau}^{\left(v_{j-1}\right)}$. Let $l_{j}^{(x)}$ be defined by equality (19), where $\hat{\tau}_{1}, \ldots, \hat{\tau}_{j-1}$ are replaced with $\hat{\tau}^{\left(v_{1}\right)}, \ldots, \hat{\tau}^{\left(v_{j-1}\right)}$ and the $\hat{\tau}_{j}$ are replaced with $\hat{\tau}^{(k)}$. Now let $v_{j}$ be the minimal number $k$ such that $k \leq m n$, $k>v_{j-1}$, conditions (32) hold, and

$$
\Delta\left(\hat{X}\left(x, \hat{\tau}^{(k)}\right)-\right), \quad p^{(k)} \in V_{\sigma}\left(l_{j}^{(k)}\right) .
$$

Again, we set $v_{1}=+\infty$ if such a value $k$ does not exist.

Next we split the set

into disjoint parts

$$
\left\{v_{m}<+\infty\right\}
$$

$$
\left\{v_{1}=k_{1}, \ldots, v_{m}=k_{m}\right\}=: \Omega_{k_{1}, \ldots, k_{m}}, \quad 1 \leq k_{1}<\cdots<k_{m} \leq m n .
$$

Given $1 \leq k_{1}<\cdots<k_{m} \leq m n$, put

$$
I_{1}=I^{\left(k_{1}\right)}, \quad \ldots, \quad I_{m}=I^{\left(k_{m}\right)}
$$

and

$$
\Xi_{k_{1}, \ldots, k_{m}}=\Omega_{k_{1}, \ldots, k_{m}} \cap\left\{\sup _{r \leq t}|\hat{X}(x, r)| \leq R\right\} .
$$

Here $\hat{X}$ denotes the solution of equation (11) for which the jump moments $\tau^{\left(v_{1}\right)}, \ldots, \tau^{\left(v_{m}\right)}$ of the process $Z$ are replaced with $\hat{\tau}^{\left(v_{1}\right)}, \ldots, \hat{\tau}^{\left(v_{m}\right)}$. 
It is clear from the construction that

$$
\Xi_{k_{1}, \ldots, k_{m}} \subset \Xi_{\mathbf{I}}, \quad \mathbf{I}=\prod_{j=1}^{m} I_{j},
$$

and that the set $\Xi_{k_{1}, \ldots, k_{m}}$ is of the form (17). For an arbitrary $y \in B_{\varkappa}(x)$, estimate (18) implies that

$$
\mu_{x, t, \Xi_{k_{1}, \ldots, k_{m}}} \wedge \mu_{y, t, \Xi_{k_{1}, \ldots, k_{m}}} \geq \delta^{m}\left(\frac{1-\gamma}{1+\gamma}\right)^{2 m} \mathrm{P}\left(\Xi_{k_{1}, \ldots, k_{m}}\right) .
$$

Note that the $\Xi_{k_{1}, \ldots, k_{m}}$ are disjoint: hence

$$
\mu_{x, t} \geq \sum_{1 \leq k_{1}<\cdots<k_{j} \leq m n} \mu_{x, t, \Xi_{k_{1}, \ldots, k_{m}}}, \quad \mu_{y, t} \geq \sum_{1 \leq k_{1}<\cdots<k_{j} \leq m n} \mu_{y, t, \Xi_{k_{1}, \ldots, k_{m}}} .
$$

Moreover

$$
\mu_{x, t, \Xi_{k_{1}, \ldots, k_{m}}}\left(\mathbb{R}^{m}\right)=\mu_{y, t, \Xi_{k_{1}, \ldots, k_{m}}}\left(\mathbb{R}^{m}\right)=\mathrm{P}\left(\Xi_{k_{1}, \ldots, k_{m}}\right) .
$$

This allows us to apply statement 2 of Lemma A.2 and to conclude that

$$
\mu_{x, t} \wedge \mu_{y, t} \geq \delta^{m}\left(\frac{1-\gamma}{1+\gamma}\right)^{2 m} \mathrm{P}\left(\bigcup_{1 \leq k_{1}<\cdots<k_{j} \leq m n} \Xi_{k_{1}, \ldots, k_{m}}\right) .
$$

Estimate (28) implies that

$$
\Omega_{k_{1}, \ldots, k_{m}} \cap\left\{\sup _{r \leq t}|X(x, r)| \leq R-\frac{m \varepsilon}{2} A_{1} \varrho e^{t A_{1}}\right\} \subset \Xi_{k_{1}, \ldots, k_{m}} .
$$

Thus

$$
\begin{gathered}
\mathrm{P}\left(\bigcup_{1 \leq k_{1}<\cdots<k_{j} \leq n} \Xi_{k_{1}, \ldots, k_{m}}\right) \\
\geq \mathrm{P}\left(\left\{v_{m}<+\infty\right\} \cap\left\{\sup _{r \leq t}|X(x, r)| \leq R-\frac{m \varepsilon}{2} A_{1} \varrho e^{t A_{1}}\right\}\right) \\
\geq \mathrm{P}\left(v_{m}<+\infty\right)-\mathrm{P}\left(\sup _{r \leq t}|X(x, r)|>R-\frac{m \varepsilon}{2} A_{1} \varrho e^{t A_{1}}\right) .
\end{gathered}
$$

Now Theorem 2.1 follows from the following auxiliary result.

\section{Lemma 3.3.}

$$
\mathrm{P}\left(v_{m}<+\infty\right) \geq \mathrm{P}_{1}
$$

Proof. It is clear that

$$
\mathrm{P}\left(v_{m}<+\infty\right) \geq \mathrm{P}\left(v_{1} \leq n, \ldots,(m-1) n<v_{m} \leq m n\right) .
$$

The probability $\mathrm{P}\left(v_{1}>n\right)$ is easy to estimate. Indeed, the random event $v_{1}>n$ is the intersection of $n$ events, where the event of index $k$ is defined as a collection of those elementary events where at least one of the conditions in (32) does not hold. Put $\mathcal{F}_{k}^{\varepsilon}=\sigma\left(Z_{s}, s \leq k \varepsilon\right)$. Given the $\sigma$-algebra $\mathcal{F}_{k-1}^{\varepsilon}$, the conditional probability that all conditions in (32) hold is not less than

$$
\begin{aligned}
\left(\varepsilon \Pi(\Gamma) e^{-\varepsilon \Pi(\Gamma)}\right) P\left(\sup _{s \in I^{(k)}} \mid Z_{2}(s)\right. & \left.-Z_{2}\left(\hat{\tau}^{(k)}\right) \mid \leq \theta\right) \\
& \times\left(\frac{\inf _{x} \Pi(u \in \Gamma:|a(x+u)-a(x)|>\rho,|u| \leq \varrho)}{\Pi(\Gamma)}\right),
\end{aligned}
$$


since the process $Z_{2}^{(k)}:=\left\{Z_{2}(s)-Z_{2}((k-1) \varepsilon), s \geq(k-1) \varepsilon\right\}$ is independent of $\mathcal{F}_{k-1}^{\varepsilon}$, the random variable $\nu^{(k)}=\nu\left(I^{(k)} \times \Gamma\right)$ is independent of $\mathcal{F}_{k-1}^{\varepsilon} \vee \sigma\left(Z_{2}^{(k)}\right)$, and $p^{(k)}$ is independent of $\mathcal{F}_{k-1}^{\varepsilon} \vee \sigma\left(Z_{2}^{(k)}, \nu^{(k)}\right)$. Moreover, $\nu^{(k)}$ is a Poisson random variable with parameter $\varepsilon \Pi(\Gamma)$, the variable $X\left(x, \hat{\tau}^{(k)}-\right)$ is measurable with respect to $\mathcal{F}_{k-1}^{\varepsilon} \vee \sigma\left(Z_{2}^{(k)}\right)$, and the distribution of $p^{(k)}$ is $\Pi(\cdot \cap \Gamma) /(\Pi(\Gamma))$.

It is clear that

$$
\begin{aligned}
& \mathrm{P}\left(\sup _{s \in I^{(k)}}\left|Z_{2}(s)-Z_{2}\left(\hat{\tau}^{(k)}\right)\right| \leq \theta\right) \\
& \geq 1-\mathrm{P}\left(\sup _{s \in\left((k-1) \varepsilon, \tau^{(k)}\right)}\left|Z_{2}(s)-Z_{2}\left(\hat{\tau}^{(k)}\right)\right|>\theta\right) \\
&-\mathrm{P}\left(\sup _{s \in\left(\tau^{(k)}, k \varepsilon\right)}\left|Z_{2}(s)-Z_{2}\left(\hat{\tau}^{(k)}\right)\right|>\theta\right) .
\end{aligned}
$$

Then we apply the Doob maximal inequality for the submartingale

$$
\left(\tau^{(k)}, k \varepsilon\right) \ni s \mapsto\left|Z_{2}(s)-Z_{2}\left(\hat{\tau}^{(k)}\right)\right|^{2}
$$

and obtain

$$
\mathrm{P}\left(\sup _{s \in\left(\tau^{(k)}, k \varepsilon\right)}\left|Z_{2}(s)-Z_{2}\left(\hat{\tau}^{(k)}\right)\right|>\theta\right) \leq \frac{1}{\theta^{2}} \mathrm{E}\left|Z_{2}(k \varepsilon)-Z_{2}\left(\hat{\tau}^{(k)}\right)\right|^{2}=\frac{\varepsilon}{2 \theta^{2}} \int_{\Gamma^{c}}|u|^{2} \Pi(d u) .
$$

On the other hand, the inequality

$$
\sup _{s \in\left((k-1) \varepsilon, \tau^{(k)}\right)}\left|Z_{2}(s)-Z_{2}\left(\hat{\tau}^{(k)}\right)\right|>\theta
$$

implies that

$$
\sup _{s \in\left((k-1) \varepsilon, \tau^{(k)}\right)}\left|Z_{2}(s)-Z_{2}((k-1) \varepsilon)\right|>\frac{\theta}{2} .
$$

Applying Doob's inequality again we prove that

$$
\mathrm{P}\left(\sup _{s \in\left((k-1) \varepsilon, \tau^{(k)}\right)}\left|Z_{2}(s)-Z_{2}\left(\hat{\tau}^{(k)}\right)\right|>\theta\right) \leq \frac{2 \varepsilon}{\theta^{2}} \int_{\Gamma^{c}}|u|^{2} \Pi(d u) .
$$

The latter bounds and the inequality $1-z \leq e^{-z}, z \geq 0$, imply that

$$
\begin{aligned}
\mathrm{P}\left(v_{1}>n\right) & \leq\left[1-\varepsilon \Pi_{1} e^{-\varepsilon \Pi(\Gamma)}\left(1-\frac{5 \varepsilon}{2 \theta^{2}} \int_{\Gamma^{c}}|u|^{2} \Pi(d u)\right)\right]^{n} \\
& \leq \exp \left(-\varepsilon n \Pi_{1} e^{-\varepsilon \Pi(\Gamma)}\left(1-\frac{5 \varepsilon}{2 \theta^{2}} \int_{\Gamma^{c}}|u|^{2} \Pi(d u)\right)\right) .
\end{aligned}
$$

Similarly we show that

$$
\begin{aligned}
& \mathrm{P}\left(v_{j}>j n, v_{j-1} \leq(j-1) n \mid \mathcal{F}_{(j-1) n}^{\varepsilon}\right) \\
& \quad \leq \exp \left(-\varepsilon n \Pi_{j} e^{-\varepsilon \Pi(\Gamma)}\left(1-\frac{5 \varepsilon}{2 \theta^{2}} \int_{\Gamma^{c}}|u|^{2} \Pi(d u)\right)\right) \mathbb{1}_{v_{j-1} \leq(j-1) n}
\end{aligned}
$$

for an arbitrary $j=2, \ldots, m$. Multiplying the latter inequalities and recalling that $n=[t /(m \varepsilon)]$ we complete the proof. 


\section{The EXPONEntial ERgODICITy FOR PROCESSES With LÉVY NOISE}

Let the symbol $\mathcal{Q}$ stand for the set of $f \in C^{2}\left(\mathbb{R}^{m}\right)$ for which the function

$$
x \mapsto \int_{|u|>1} f(x+u) \Pi(d u)
$$

is locally bounded. For $f \in \mathcal{Q}$, put

$$
\begin{gathered}
\mathcal{A} f(x)=(\nabla f(x), a(x)+b)+\int_{\mathbb{R}^{m}}\left[f(x+u)-f(x)-(\nabla f(x), u) \mathbb{1}_{|u| \leq 1}\right] \Pi(d u), \\
x \in \mathbb{R}^{m} .
\end{gathered}
$$

The following result is proved in [1] (see Theorem 1.1 therein). For a given probability measure $\mu$ in $\mathbb{R}^{m}$, put

$$
\mu_{s}(d y)=\int_{\mathbb{R}^{m}} P_{s}(x, d y) \mu(d x), \quad s>0 .
$$

Theorem 4.1. Assume that the following two conditions hold.

1. For an arbitrary $R>0$, there exists $T=T(R)$ such that

$$
\delta(T, R):=\inf _{|x|,|y| \leq R}\left(\mu_{x, T} \wedge \mu_{y, T}\right)>0 .
$$

2. There exist a function $\varphi \in \mathcal{Q}$ and constants $\alpha>0$ and $\beta>0$ such that

$$
\begin{aligned}
& \qquad \mathcal{A} \varphi \leq-\alpha \varphi+\beta \\
& \text { and } \varphi(x) \rightarrow+\infty \text { as }|x| \rightarrow \infty . \\
& \text { Then the process } X \text { has a unique invariant distribution } \pi \text {. Moreor } \mathrm{C}_{1} \text { and } \mathrm{C}_{2} \text { such that } \\
& \text { positive constants } \\
& \quad\left\|\mu_{s}-\pi\right\|_{\text {var }} \leq\left(1+\int_{\mathbb{R}^{m}} \varphi d \mu\right) e^{\mathrm{C}_{1}-\mathrm{C}_{2} s}, \quad s \in \mathbb{R}^{+},
\end{aligned}
$$

Then the process $X$ has a unique invariant distribution $\pi$. Moreover, there exist

for an arbitrary probability measure $\mu$.

Explicit expressions for the constants $C_{1}$ and $C_{2}$ are given in the proof of Theorem 1.1 of the paper [1]. These constants are expressed in terms of the function $\varphi$ and variable $\delta(T, R)$. Our aim is to provide similar expressions in terms of the Lévy measure $\Pi$ of the process $Z$ in equation (1) and the coefficient $a$ of this equation.

Lyapunov's condition (33) is easy to check in this case; see Section 4.1 in [1] and the references therein.

Theorem 2.1, the Markov property of the process $X$, and Dobrushin's lemma (see Appendix Amply the following bound for $\delta(T, R)$ with some $T$ and $R$.

Lemma 4.1. Assume that all the assumptions of Theorem 2.1 hold. If for given $t_{1}>0$ and $R_{1}>0$

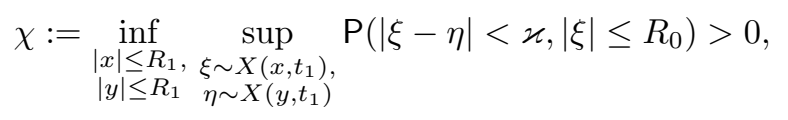

then

$$
\delta\left(t+t_{1}, R_{1}\right) \geq \chi \delta^{m}\left(\frac{1-\gamma}{1+\gamma}\right)^{2 m}\left(\mathrm{P}_{1}-\mathrm{P}_{2}\right) .
$$

The proof of Lemma 4.1 is similar to that of Lemma 3.2 in [1]; thus we omit it here. 
Remark 4.1. Inequality (35) follows, for example, from condition $\mathbf{S}$ of the paper [1]; condition $\mathbf{S}$ can effectively be checked in terms of the process $Z$ and coefficient $a$ of equation (11) (see Section 4.3 in [1]). Other possibilities to check inequality (35) are available if one imposes some additional assumptions concerning the equation (see Section 5 , where (35) follows from the dissipativity condition (38) ).

Substituting the bound from the preceding Lemma 4.1 in the explicit expressions for the constants $C_{1}$ and $C_{2}$ given in the proof of Theorem 1.1 of the paper [1, we obtain the following result.

Theorem 4.2. Assume that all assumptions of Theorem 2.1 and assumption 2 of Theorem 4.1 hold. Assume further that relation (35) is satisfied for given $c \in(0,1), t_{1}>0$, and $R_{1}>0$. Finally we assume that

$$
\varphi(x)+\varphi(y) \geq \max \left(\frac{2 \beta}{c \alpha}, 1\right), \quad \max (|x|,|y|) \geq R_{1} .
$$

Put

$$
\begin{gathered}
T=t+t_{1}, \quad \varsigma=\chi \delta^{m}\left(\frac{1-\gamma}{1+\gamma}\right)^{2 m}\left(\mathrm{P}_{1}-\mathrm{P}_{2}\right) \\
Q=\frac{1}{\ln (1 / \varsigma)}\left(\frac{(1-c) \alpha}{2} T+\ln \left(1+\frac{4 \beta}{\alpha}+4 \sup _{|x| \leq R_{1}} \varphi(x)\right)\right) .
\end{gathered}
$$

Then inequality (34) holds with

$$
\begin{gathered}
\mathrm{C}_{1}=\frac{(1-c) \alpha T}{2}+\max \left(\ln \left(\frac{\beta}{\alpha}\right), 0\right)+\ln \left(\frac{4}{1-(1-\varsigma)^{1 / 2}}\right), \\
\mathrm{C}_{2}=\frac{(1-c) \alpha}{4 \max (Q, 1)} .
\end{gathered}
$$

\section{EXAMPLE}

Let the process $Z(t)$ in equation (11) be of the form

$$
Z(t)=\int_{0}^{t} \int_{\mathbb{R}^{2}} u \tilde{\nu}(d s, d u),
$$

where the Lévy measure $\Pi$ is such that

$$
\int_{\mathbb{R}^{2}}|u|^{2} \Pi(d u)<+\infty .
$$

Let the function $a$ satisfy the following dissipativity condition: $a(0)=0$ and there exists a constant $A_{3}>0$ such that

$$
(x-y, a(x)-a(y)) \leq-A_{3}|x-y|^{2}
$$

for all $x, y \in \mathbb{R}^{2}$.

We prove, under conditions (37) and (38), that the solution of equation (11) satisfies all the assumptions of Theorem 4.2, find explicit values of the constants $\alpha$ and $\beta$, and derive a lower bound for $\varsigma$.

We have for the function $f(x)=|x|^{2}, x \in \mathbb{R}^{2}$, that

$$
\mathcal{A} f(x)=2(x, a(x))+\int_{\mathbb{R}^{2}}|u|^{2} \Pi(d u) .
$$


Condition (38) implies

$$
\mathcal{A} f(x) \leq-2 A_{3}|x|^{2}+\int_{\mathbb{R}^{2}}|u|^{2} \Pi(d u),
$$

that is, (33) holds with $\alpha=2 A_{3}$ and $\beta=\int_{\mathbb{R}^{2}}|u|^{2} \Pi(d u)$.

Next we find the estimates for $\mathrm{P}_{2}$ and $\chi$. Put $q_{1}=R-\frac{1}{2} m \varepsilon A_{1} \varrho e^{t A_{1}}$. Then

$$
\begin{aligned}
\mathrm{P}_{2} & =\sup _{|x| \leq R_{0}} \mathrm{P}\left(\sup _{r \leq t}|X(x, r)|>q_{1}\right) \\
& \leq \sup _{|x| \leq R_{0}}\left[\mathrm{P}\left(\sup _{r \leq t}|Z(t)|>\frac{q_{1}}{2}\right)+\mathrm{P}\left(\sup _{r \leq t}\left|x+\int_{0}^{r} a(X(x, s)) d s\right|>\frac{q_{1}}{2}\right)\right] .
\end{aligned}
$$

By assumption, $Z$ is a martingale, that is, $|Z|$ is a submartingale. Thus Doob's maximal inequality implies

$$
\mathrm{P}\left(\sup _{r \leq t}|Z(t)|>\frac{q_{1}}{2}\right) \leq \frac{4}{q_{1}^{2}} \mathrm{E}|Z(t)|^{2}=\frac{4 t}{q_{1}^{2}} \int_{\mathbb{R}^{2}}|u|^{2} \Pi(d u)=\frac{4 \beta t}{q_{1}^{2}} .
$$

Consider the function

$$
g(r)=\int_{0}^{r}|a(X(x, s))| d s, \quad r \leq t .
$$

The function $a$ satisfies the linear growth condition:

$$
|a(x)| \leq A_{1}|x| .
$$

Hence

$$
\begin{aligned}
g(r) & \leq A_{1} \int_{0}^{r}|X(x, s)| d s=A_{1} \int_{0}^{r}\left|x+\int_{0}^{s} a(X(x, u)) d u+Z(s)\right| d s \\
& \leq A_{1} \int_{0}^{r}(|x|+g(s)) d s+A_{1} \int_{0}^{t}|Z(s)| d s .
\end{aligned}
$$

The Gronwall lemma implies that

$$
g(r) \leq\left[A_{1}|x| r+A_{1} \int_{0}^{t}|Z(s)| d s\right] e^{r A_{1}} .
$$

Then

$$
\sup _{r \leq t} g(r) \leq\left[|x| t+\int_{0}^{t}|Z(s)| d s\right] A_{1} e^{t A_{1}} .
$$

We consider the case of $|x| \leq R_{0}$. Then

$$
\begin{gathered}
\mathrm{P}\left(\sup _{r \leq t}\left|x+\int_{0}^{r} a(X(x, s)) d s\right|>\frac{q_{1}}{2}\right) \leq \mathrm{P}\left(\sup _{r \leq t} g(r)>\frac{q_{1}}{2}-|x|\right) \\
\quad \leq \mathrm{P}\left(\int_{0}^{t}|Z(s)| d s>\frac{1}{A_{1}}\left(\frac{q_{1}}{2}-R_{0}\right) e^{-t A_{1}}-R_{0} t\right) \leq \frac{1}{q_{2}^{2}} \mathrm{E}\left(\int_{0}^{t}|Z(s)| d s\right)^{2} \\
\quad \leq \frac{t}{q_{2}^{2}} \int_{0}^{t} \mathrm{E}|Z(s)|^{2} d s=\frac{\beta t^{3}}{2 q_{2}^{2}}
\end{gathered}
$$

where

$$
q_{2}=\frac{1}{A_{1}}\left(\frac{q_{1}}{2}-R_{0}\right) e^{-t A_{1}}-R_{0} t .
$$

Thus

$$
\mathrm{P}_{2} \leq \beta t\left(\frac{4}{q_{1}^{2}}+\frac{t^{2}}{2 q_{2}^{2}}\right)
$$


and

$$
\begin{aligned}
\chi & =\inf _{\substack{|x| \leq R_{1},|y| \leq R_{1}}} \sup _{\substack{\xi \sim X\left(x, t_{1}\right), \eta \sim X\left(y, t_{1}\right)}} \mathrm{P}\left(|\xi-\eta|<\varkappa,|\xi| \leq R_{0}\right) \\
\geq & \inf _{\substack{|x| \leq R_{1},|y| \leq R_{1}}} \sup _{\substack{\xi \sim X\left(x, t_{1}\right), \eta \sim X\left(y, t_{1}\right)}}\left[1-\mathrm{P}(|\xi-\eta| \geq \varkappa)-\mathrm{P}\left(|\xi|>R_{0}\right)\right] \\
\geq & \inf _{\substack{|x| \leq R_{1},|y| \leq R_{1}}}\left[1-\mathrm{P}\left(\left|X\left(x, t_{1}\right)-X\left(y, t_{1}\right)\right| \geq \varkappa\right)-\mathrm{P}\left(\left|X\left(x, t_{1}\right)\right|>R_{0}\right)\right] \\
\geq & 1-\sup _{\substack{|x| \leq R_{1},|y| \leq R_{1}}} \mathrm{P}\left(\left|X\left(x, t_{1}\right)-X\left(y, t_{1}\right)\right| \geq \varkappa\right)-\sup _{|x| \leq R_{1}} \mathrm{P}\left(\left|X\left(x, t_{1}\right)\right|>R_{0}\right) .
\end{aligned}
$$

Condition (38) implies that the random variable $\psi_{x, y}\left(t_{1}\right)=\left|X\left(x, t_{1}\right)-X\left(y, t_{1}\right)\right|^{2}$ admits the bound

$$
\psi_{x, y}\left(t_{1}\right) \leq \psi_{x, y}(0) e^{-2 t_{1} A_{3}}=|x-y|^{2} e^{-2 t_{1} A_{3}} .
$$

Hence

$$
\mathrm{P}\left(\left|X\left(x, t_{1}\right)-X\left(y, t_{1}\right)\right| \geq \varkappa\right) \leq \mathrm{P}\left(|x-y| e^{-A_{3} t_{1}} \geq \varkappa\right)=\mathbb{1}_{|x-y| e^{-t_{1} A_{3}} \geq \varkappa} .
$$

The Chebyshev inequality and Lemma 3.3 of the paper [1] imply that

$$
\sup _{|x| \leq R_{1}} \mathrm{P}\left(\left|X\left(x, t_{1}\right)\right|>R_{0}\right) \leq \frac{1}{R_{0}^{2}}\left(\frac{\beta}{\alpha}+R_{1}^{2} e^{-t_{1} \alpha}\right) .
$$

This gives a lower bound for $\chi$ :

$$
\chi \geq \mathbb{1}_{2 R_{1} e^{-t_{1} A_{3}<\varkappa}}-\frac{1}{R_{0}^{2}}\left(\frac{\beta}{\alpha}+R_{1}^{2} e^{-t_{1} \alpha}\right)
$$

and, as a result, a bound for $\varsigma$ :

$$
\varsigma \geq\left(\mathbb{1}_{2 R_{1} e^{-t_{1} A_{3}<\varkappa}}-\frac{1}{R_{0}^{2}}\left(\frac{\beta}{\alpha}+R_{1}^{2} e^{-t_{1} \alpha}\right)\right) \delta^{m}\left(\frac{1-\gamma}{1+\gamma}\right)^{2 m}\left(\mathrm{P}_{1}-\beta t\left(\frac{4}{q_{1}^{2}}+\frac{t^{2}}{2 q_{2}^{2}}\right)\right) .
$$

Below we exhibit the above general results and find explicitly the constants $C_{1}$ and $C_{2}$ for a particular function $a$, Lévy measure $\Pi$, and for some constants involved in conditions (5) and (6) of Theorem 2.1, in condition (33) of Theorem 4.1, and in condition (36) of Theorem 4.2 .

Let $a(x)=-x, x \in \mathbb{R}^{2}$. Then $A_{0}=A_{2}=0$ and $A_{1}=A_{3}=1$. Let the Lévy measure $\Pi$ be concentrated in the ring $\Gamma=\left\{u \in \mathbb{R}^{2}|1<| u \mid \leq 2\right\}$ and let $\Pi(\Gamma)=\Pi_{1}=5$. One can put $\rho=1$ and $\varrho=2$. We also assume that $\int_{\mathbb{R}^{2}}|u|^{2} \Pi(d u)=10$ and that $\Pi_{2}=1$ for $\sigma=\frac{1}{\sqrt{2}}$.

The above results yield $\alpha=2$ and $\beta=10$ in the case under consideration. Since $\Pi\left(\Gamma^{c}\right)=0$, we get $\theta=0$. Now we choose $R_{1}$ and $c$ such that condition (36) holds, namely $R_{1}=5$ and $c=1 / 2$. Put $t=1$ and $R_{0}=4$. In order that the right-hand side of inequality (40) be positive for large $t_{1}$, it is necessary that $R_{0}^{2}>\beta / \alpha=5$. Let $R$ be such that $\mathrm{P}_{1,0}>\mathrm{P}_{2}$, namely $R=60$. Put $\delta=\gamma=1 / 2$. Then we choose $\varepsilon$ satisfying condition (15), namely $\varepsilon=10^{-5}$. After this we choose $\varkappa$ satisfying condition (6) , namely $\varkappa=5 \cdot 10^{-9}$. Finally, we choose $t_{1}$ such that the right-hand side of inequality (40) is positive, namely $t_{1}=22$. Then the constants $C_{1}$ and $C_{2}$ are such that

$$
\mathrm{C}_{1}=22.97918651, \quad \mathrm{C}_{2}=0.11936229 .
$$




\section{Appendix A. The COMmon part of MeAsures}

The basic property of the common part of measures (3) is described in the following result widely known as Dobrushin's lemma; see [4].

Lemma A.1. Let $\mu$ and $\kappa$ be probability measures in a complete separable metric space $\mathbb{X}$. Then

$$
\mu \wedge \kappa=\max _{\xi \sim \mu, \eta \sim \kappa} \mathrm{P}(\xi=\eta) .
$$

In other words, if two arbitrary random elements $\xi$ and $\eta$ assume values in $\mathbb{X}$ and have distributions $\mu$ and $\kappa$, respectively, then

$$
\mathrm{P}(\xi=\eta) \leq \mu \wedge \kappa
$$

and there are some probability space and random elements $\xi$ and $\eta$ defined in this probability space for which inequality (41) becomes an equality.

The following results are consequences of Lemma A.1

Lemma A.2. 1. Let $\mu$ and $\kappa$ be finite measures in a complete separable metric space $\mathbb{X}$ such that $\mu(\mathbb{X})=\kappa(\mathbb{X})$. Let $F: \mathbb{X} \rightarrow \mathbb{Y}$ be a measurable mapping. Then

$$
\left(\mu \circ F^{-1}\right) \wedge\left(\kappa \circ F^{-1}\right) \geq \mu \wedge \kappa .
$$

2. Let $\mu_{k}, \kappa_{k}, k=1, \ldots, n$, be finite measures concentrated in $\mathbb{X}_{k}, k=1, \ldots, n$, respectively. Assume that $\mu_{k}(\mathbb{X})=\kappa_{k}(\mathbb{X})$. Then

$$
\left(\sum_{k} \mu_{k}\right) \wedge\left(\sum_{k} \kappa_{k}\right) \geq \sum_{k} \mu_{k} \wedge \kappa_{k} .
$$

3. Let

$$
\mu=\int_{\Theta} \mu_{\theta}(\cdot) \pi(d \theta), \quad \kappa=\int_{\Theta} \kappa_{\theta}(\cdot) \pi(d \theta)
$$

where $\pi$ is a finite measure in a measure space $\Theta$ and where $\mu_{\theta}(A)$ and $\kappa_{\theta}(A)$ are measurable functions with respect to the variable $\theta$ and are probability measures with respect to the variable $A$.

Then

$$
\mu \wedge \kappa \geq \int_{\Theta}\left(\mu_{\theta} \wedge \kappa_{\theta}\right) \pi(d \theta) .
$$

Statements 1 and 2 follow directly from Dobrushin's lemma. The proof of statement 3 also relies on Dobrushin's lemma but uses additional technical reasoning. In the proof of statement 3 , one needs to find a family of pairs $\left(\xi_{\theta}, \kappa_{\theta}\right)$ for given families of measures $\left\{\mu_{\theta}\right\}$ and $\left\{\kappa_{\theta}\right\}$ such that the inequality for $\mu_{\theta} \wedge \kappa_{\theta}$ (similar to (41)) becomes an equality. Moreover, the family of pairs $\left(\xi_{\theta}, \kappa_{\theta}\right)$ should necessarily be measurable with respect to $\theta$. The existence of such a family follows from the "three random variables lemma"; see [5].

\section{APPENDIX B. IMPLICIT FUNCTION THEOREM}

Let $U$ be an open domain in $\mathbb{R}^{m} \times \mathbb{R}^{m}$. Put $U_{x}=\{z \mid(x, z) \in U\}$ and let $\nabla_{k}$ be the derivative with respect to the $k$-th variable.

Theorem B.1. Let a continuous function $F: U \rightarrow \mathbb{R}^{m}$ satisfy the following conditions.

1) The Sobolev derivative

$$
\nabla_{z} F(x, z)=\left(\frac{\partial}{\partial_{z_{1}}} F(x, z), \ldots, \frac{\partial}{\partial_{z_{m}}} F(x, z)\right)
$$

exists in the domain $U$. 
2) There are a constant $\gamma \in(0,1)$ and a nonsingular matrix $H$ such that the inequality

$$
\left\|\nabla_{z} F(x, z)-H\right\| \leq \gamma\left\|H^{-1}\right\|^{-1}
$$

holds for all $x$ and almost everywhere with respect to $z$ such that $(x, z) \in U$.

3) The derivative $\nabla_{x} F(x, z)$ exists in the usual sense and moreover

$$
\left\|\nabla_{x} F(x, z)\right\| \leq D
$$

for all $(x, z) \in U$.

Further let constants $\varkappa>0$ and $r>0$ be such that

4) $D\left\|H^{-1}\right\| \varkappa \leq(1-\gamma) r$.

Finally let $V_{r}=\left\{z \mid \bar{B}_{r}(z) \subset U_{x}\right\}$. Then there exists a unique function $K: B_{\varkappa}(x) \times$ $V_{r} \rightarrow \mathbb{R}^{m}$ such that

$$
F(y, K(y, z))=F(x, z)
$$

for all $y \in B_{\varkappa}(x)$ and $z \in V_{r}$.

Below we list some of the properties of the function $K$ :

(1) The Sobolev derivative $\nabla_{z} K(y, z)$ exists for all $y \in B_{\varkappa}(x)$ and $z \in V_{r}$; moreover

$$
\nabla_{z} K(y, z)=\left[\nabla_{2} F(y, K(y, z))\right]^{-1} \nabla_{z} F(x, z) .
$$

(2) The function $K(y, z)$ satisfies the Lipshitz condition with respect to the variable $z$ for an arbitrary $y \in B_{\varkappa}(x)$.

(3) The following two-sided bound holds for all $y \in B_{\varkappa}(x)$ and $z \in V_{r}$ :

$$
\left(\frac{1-\gamma}{1+\gamma}\right)^{m} \leq\left|\operatorname{det} \nabla_{z} K(y, z)\right| \leq\left(\frac{1+\gamma}{1-\gamma}\right)^{m} \text {. }
$$

The proof of Theorem B.1 is similar to that of Theorem 2.2.7 in [6]. Property (1) follows from the proof of Theorem B.1, while property (2) is derived from the boundedness of the derivative (44). Bound (45) is a corollary of (42), (44), and the inequality

$$
|\operatorname{det} A| \leq\|A\|^{m},
$$

where $A$ is an $m \times m$ matrix. The latter inequality is proved by reducing the matrix $A$ to the normal Jordan norm and by using the property of the spectral radius of a matrix (see, for example, Theorem 6.1.3 in [7]).

\section{BIBLIOGRAPHY}

1. A. M. Kulik, Exponential ergodicity of the solutions to SDE's with a jump noise, Stoch. Process. Appl. 119 (2009), no. 2, 602-632. MR2494006 (2010i:60176)

2. A. M. Kulik, Absolute continuity and convergence in variation for distributions of functionals of Poisson point measure, J. Theor. Probab. 24 (2011), no. 1, 1-38. MR2782709 (2012e:60154)

3. G. Federer, Geometric Measure Theory, Springer, New York, 1969. MR0257325 (41:1976)

4. R. L. Dobrushin, Prescribing a system of random variables by conditional distributions, Teor. Veroyatnost. Primenen. 15 (1970), no. 3, 469-497; English transl. in Theory Probab. Appl. 15 (1970), no. 3, 458-486. MR0298716 (45:7765)

5. A. Yu. Veretennikov, Coupling method for Markov chains under integral Doeblin type condition, Theory Stoch. Process. 8(24) (2002), no. 3-4, 383-391. MR2027410 (2004j:60151)

6. L. Nierenberg, Topics in Nonlinear Functional Analysis, Courant Institute of Mathematical Sciences, New York University, New York, 1974. MR0488102 (58:7672)

7. P. Lankaster, Theory of Matrices, Academic Press, New York-London, 1969. MR0245579 (39:6885) 
National Technical University of Ukraine "KPI", Peremogy Avenue 37, 03056, Kyiv, UKRAINE

E-mail address: sem_bodn@ukr.net

Institute of Mathematics, National Academy of Science of Ukraine, Tereshchenkivs'Ka Street 3, 01601, Kyiv, Ukraine

E-mail address: kulik@imath.kiev.ua

Received 05/DEC/2011

Translated by N. SEMENOV 